\title{
Baseline Sensitivity of Fusarium virguliforme to Fluopyram Fungicide
}

\author{
Jie Wang, Department of Plant, Soil and Microbial Sciences, Michigan State University, East Lansing 48824; Carl A. Bradley, Department of \\ Crop Sciences, University of Illinois, Urbana 61801; Olivia Stenzel, Department of Plant, Soil and Microbial Sciences, Michigan State Uni- \\ versity, East Lansing 48824; Dianne K. Pedersen and Ursula Reuter-Carlson, Department of Crop Sciences, University of Illinois, Urbana \\ 61801; and Martin I. Chilvers, Department of Plant, Soil and Microbial Sciences, Michigan State University, East Lansing 48824
}

\begin{abstract}
Fluopyram, a succinate dehydrogenase inhibitor (SDHI) fungicide, was recently registered for use as a soybean seed treatment for management of sudden death syndrome (SDS) caused by Fusarium virguliforme. Although registered and now used commercially, in vitro baseline fungicide sensitivity of $F$. virguliforme to fluopyram has not yet been established. In this study, the baseline sensitivity of $F$. virguliforme to fluopyram was determined using in vitro growth of mycelium and germination of conidia assays with two collections of $F$. virguliforme isolates. A total of 130 and $75 \mathrm{~F}$. virguliforme isolates were tested using the mycelial growth and conidia germination assays, respectively, including a core set of isolates that were tested with both assays. In the mycelial growth inhibition assay, 113 out of 130 isolates $(86.9 \%)$ were inhibited $50 \%$ by effective concentrations $\left(\mathrm{EC}_{50}\right)$ less than $5 \mu \mathrm{g} / \mathrm{ml}$ with a mean $\mathrm{EC}_{50}$ of $3.35 \mu \mathrm{g} / \mathrm{ml}$. For the conidia germination assay, 73 out of 75 isolates (97\%) were determined to

have an estimated $\mathrm{EC}_{50}$ of less than $5 \mu \mathrm{g} / \mathrm{ml}$ with a mean $\mathrm{EC}_{50}$ value of $2.28 \mu \mathrm{g} / \mathrm{ml}$. In a subset of 20 common isolates that were phenotyped with both assays, conidia germination of $F$. virguliforme was determined to be more sensitive to fluopyram (mean $\mathrm{EC}_{50}=2.28 \mu \mathrm{g} / \mathrm{ml}$ ) than mycelial growth (mean $\left.\mathrm{EC}_{50}=3.35 \mu \mathrm{g} / \mathrm{ml}\right)$. Hormetic effects were observed in the mycelial growth inhibition assay as $22 \%$ of the isolates demonstrated more growth on medium amended with the lowest fluopyram concentration $(1 \mu \mathrm{g} / \mathrm{ml})$, as compared with the nonfluopyram amended control. It was not possible to determine $\mathrm{EC}_{50}$ values for nine out of 185 isolates (4.8\%), as those isolates were not inhibited by $50 \%$ even at the highest fluopyram concentrations of $100 \mu \mathrm{g} / \mathrm{ml}$ for mycelial growth and $20 \mu \mathrm{g} / \mathrm{ml}$ for conidia germination inhibition assays. On the whole, the $F$. virguliforme population appears to be sensitive to fluopyram, and this study enables future monitoring of fungicide sensitivity.
\end{abstract}

Sudden death syndrome (SDS) is a major yield limiting disease in soybean (Glycine max) production. SDS is primarily caused by Fusarium virguliforme within North America (Aoki et al. 2005; O'Donnell et al. 2010). Crop rotation with common field crops, especially corn, is ineffective in reducing SDS, due to the broad host range of $F$. virguliforme (Aoki et al. 2005; Kolander et al. 2012; Navi and Yang 2016). Increased soil porosity or reduced soil moisture through tillage have been reported to reduce SDS severity (Vick et al. 2003); however, consistency of response, long term benefit, and associated costs are still not clear (Hartman et al. 2015). Planting partially resistant soybean cultivars can significantly reduce foliar SDS severity (Njiti et al. 1997). However, F. virguliforme colonization of partially resistant soybean cultivars may not be significantly different from those of susceptible cultivars (Wang et al. 2013), possibly resulting in yield loss with no obvious aboveground symptoms and enabling maintenance of $F$. virguliforme inoculum in the soil. Thus, there is a critical need to develop additional SDS disease management tools, including protection from root rot and root colonization.

Soybean fungicide seed treatments are primarily used to manage soilborne pathogens that cause seed rot, damping-off, seedling blight, and root rot (Mueller et al. 2013; Munkvold 2009). Although SDS is known for its typical foliar symptoms late in the season, root

Current address of C.A. Bradley: Department of Plant Pathology, University of Kentucky Research and Education Center, Princeton, KY 42445.

Corresponding authors: Carl A. Bradley; E-mail: carl.bradley@uky.edu; and Martin I. Chilvers; E-mail: chilvers@msu.edu

*The $\boldsymbol{e}$-Xtra logo stands for "electronic extra" and indicates that two supplementary figures and two supplementary tables are published online.

Accepted for publication 18 December 2016.

This article is in the public domain and not copyrightable. It may be freely reprinted with customary crediting of the source. The American Phytopathological Society, 2017. infections by $F$. virguliforme are essential for the development of SDS symptoms. Fusarium virguliforme can infect soybean roots shortly after seed germination (Gao et al. 2006). Early infection events also appear to be essential for severe SDS symptom development (Navi and Yang 2008). Therefore, an effective method to inhibit early infection may assist in SDS management. Fungicide seed treatments have been examined for their management of soybean SDS with only fluopyram being effective (Kandel et al. 2016; Mueller et al. 2011; Wang et al. 2014; Weems et al. 2015). However, the baseline in vitro sensitivity of $F$. virguliforme to fluopyram has not yet been determined.

Fluopyram is a succinate dehydrogenase inhibitor (SDHI) fungicide that targets the ubiquinone binding site to block energy metabolism in mitochondria (Kuhn 1984). The SDHI fungicides have been successfully utilized to manage a broad range of plant pathogens causing diseases on various fruits and vegetables (Avenot et al. 2008; Vega and Dewdney 2015). The SDHI fungicides are categorized by the Fungicide Resistance Action Committee (FRAC) to have a medium to high risk for the selection of SDHI-resistant fungal isolates (FRAC 2015). This medium to high risk is due to the single-site mode of action of SDHI fungicides, and many registered SDHI fungicides are in the same cross-resistance group (Avenot and Michailides 2010; FRAC 2015). Examples of resistance to SDHI fungicides (carboxin, fluotolanil, and boscalid) were reported shortly after their introduction to the market and have been linked to point mutations in one subunit of succinate dehydrogenase coding genes (Avenot et al. 2008; Broomfield and Hargreaves 1992; Gunatilleke et al. 1975). The recently developed SDHI fungicide fluopyram showed a lack of cross-resistance to the other SDHI fungicideresistant fungal strains (Amiri et al. 2014; Ishii et al. 2011). The low cross-resistance feature of fluopyram was demonstrated at the molecular level, by showing that the fluopyram molecule binds to a different cavity on the succinate dehydrogenase molecule and requires a lower binding energy than other SDHI fungicides (Fraaije et al. 2012).

The fungicide testing method affects the estimation of fungal sensitivity (Vega and Dewdney 2015). Fungicide-amended agar medium for the characterization of fungal mycelial growth inhibition is one of the most common methods to determine fungicide sensitivity (Liang et al. 2015; Saville et al. 2015). Spore germination rates on fungicide-amended agar medium have also been used to calculate 
fungicide sensitivities (Bradley and Pedersen 2011; Chiocchio et al. 2000). SDHI fungicides have been reported to have species-specific differential inhibition effects on mycelial growth and conidia germination (Vega and Dewdney 2015). Therefore, both mycelial growth and spore germination assays were included in this study to obtain a complete evaluation of in vitro $F$. virguliforme sensitivity to fluopyram.

To determine the efficacy of fluopyram for inhibiting growth of mycelium or germination of conidia against $F$. virguliforme, and to prolong the product life of fluopyram, it is essential to monitor for shifts in sensitivity by conducting an in vitro fungicide baseline sensitivity study. Therefore, the objectives of this study were: (i) to determine the baseline sensitivity of $F$. virguliforme against the SDHI fungicide fluopyram; and (ii) to compare the inhibition efficacy of fluopyram on $F$. virguliforme mycelia growth and conidia germination rate.

\section{Materials and Methods}

Fungal isolation collection and storage. A total of $130 \mathrm{~F}$. virguliforme isolates were tested in a mycelial growth-inhibition assay at Michigan State University (MSU). Isolates were recovered from soybean roots showing typical SDS foliar and root symptoms from 2009 to 2014 from five states (Table 1 and Supplementary Table S1): eight Arkansas isolates, 13 Illinois isolates, 11 Kansas isolates, 80 Michigan isolates, and 18 Indiana isolates. The conidia germination fungicide sensitivity assay was conducted on a collection of $75 F$. virguliforme isolates at the University of Illinois. These isolates were collected from eight states (Table 2): three Arkansas isolates, 48 Illinois isolates, three Indiana isolates, five Iowa isolates, one Kansas isolate, one Kentucky isolate, 10 Michigan isolates, one Minnesota isolate, and three from unknown sources.

All isolates were derived from a single-conidium-derived culture. Isolates at MSU were selected to ensure genetic diversity by genotyping with microsatellite markers (Wang and Chilvers 2016; Wang and Chilvers, unpublished data). Isolates were stored by allowing mycelia to colonize pieces of sterile Whatman \#1 qualitative filter paper, which was placed on the surface of potato dextrose agar (PDA) (Acumedia, Lansing, MI) in a petri plate. Once filter papers were fully colonized with mycelia, filter papers were taken from the medium, and air dried for storage at $-20^{\circ} \mathrm{C}$. Additionally, macroconidia suspensions were also added to equal volume of $30 \%$ glycerol for long-term storage at $-80^{\circ} \mathrm{C}$. Isolates used at MSU were identified to be $F$. virguliforme using a species-specific PCR assay (Wang et al. 2015).

Determination of baseline $\mathrm{EC}_{50}$ values - mycelial growth inhibition assay. The inhibition effect of fluopyram on $F$. virguliforme mycelia growth was evaluated using fungicide amended half-strength PDA (Acumedia). Formulated fluopyram (Luna Privilege; Bayer CropScience, Research Triangle Park, NC) (containing $43 \%$ active ingredient) was used to prepare stock solutions at concentrations of $1,5,10,25,50$, and $100 \mathrm{mg} / \mathrm{ml}$ (active ingredient) in sterilized water. One liter of culture medium included $12 \mathrm{~g}$ of potato dextrose broth powder (Acumedia), $15 \mathrm{~g}$ of agar (Sigma Aldrich, St. Louis, MO), and 1 liter of deionized water $\left(\mathrm{dH}_{2} \mathrm{O}\right)$. After the medium was autoclaved and cooled to $55^{\circ} \mathrm{C}, 1 \mathrm{ml}$ of fluopyram stock solution was added to the medium to make a final concentration of

Table 1. $\mathrm{EC}_{50}$ of $F$. virguliforme isolates used in the mycelial growth inhibition assay against the SDHI fungicide, fluopyram. A total of 130 isolates were collected from five states in the United States from 2009 to $2014^{\mathrm{a}}$

\begin{tabular}{lclc}
\hline State & $\mathbf{N}$ & \multicolumn{1}{c}{ Years } & $\mathbf{E C}_{\mathbf{5 0}} \mathbf{b}(\boldsymbol{\mu g} / \mathbf{m l})$ \\
\hline Arkansas & 8 & 2012 & $2.34-4.07^{*}$ \\
Illinois & 13 & $2013-2014$ & $2.15-6.92$ \\
Kansas & 11 & 2012 & $1.76-3.73^{*}$ \\
Michigan & 80 & $2009-2013$ & $1.53-9.28^{*}$ \\
Indiana & 18 & 2012 & $2.22-4.47^{*}$ \\
Total & $130^{*}$ & &
\end{tabular}

a An asterisk (*) indicates values that do not include the isolates from Arkansas $(\mathrm{n}=1)$, Kansas $(\mathrm{n}=2)$, Michigan $(\mathrm{n}=10)$, and Indiana $(\mathrm{n}=1)$ that did not reach $50 \%$ growth inhibition at the highest concentration of fluopyram $(100 \mu \mathrm{g} / \mathrm{ml})$ or nonsignificant $\mathrm{EC}_{50}$ estimation at nonlinear regression.

${ }^{\mathrm{b}}$ Range of $\mathrm{EC}_{50}$ estimation for the isolates from each state.
$0,1,5,10,25,50$, and $100 \mu \mathrm{g} / \mathrm{ml}$ (ppm) fluopyram. Isolates were divided into 11 sets tested in different experimental runs. To validate the reproducibility of this experiment, two isolates from each set of the experiment were randomly selected to verify the reproducibility of this assay (Supplementary Figure S1).

Mycelia plugs ( $2 \mathrm{~mm}^{3}$ cubes) cut from the edge of 10 day-old fungal colonies were placed mycelia side down on the center of each petri dish plate. Three replicate plates were used for each fungicide concentration. Plates were incubated at $24^{\circ} \mathrm{C}$ for 10 days in the dark, and a culture of each isolate was scanned at 3 and 10 days after inoculation (DAI) with 300 dpi image quality, using a Perfection V600 scanner (EPSON, Long Beach, CA). On each scanned image, a photographic reference scale (http://web.ncf.ca/jim/scale/) was included to calibrate the ratio between image digital pixel and physical length for the subsequent image analysis. A dark blue background card (hex color code \#1E2E4D; Hobby Lobby, Oklahoma City, OK) was used at the scanning step to create strong contrast to facilitate image analysis.

Image analysis and model selection. All images were analyzed using Assess v2.0 software (American Phytopathological Society, St. Paul, MN) using the manual panel option, and the HSV color space was used to analyze the selected colony area using a hue value between 0 and 195 . Relative mycelial growth rate was calculated by subtracting the three DAI colony areas from the 10 DAI colony area and divided by seven days to get the colony area increase per day. The growth rate of the colony area was converted to calculate the mycelial growth rate $(\mathrm{mm} / \mathrm{d})$. The R (R Core Team 2015) package "drc" (Ritz and Streibig 2005) was used to select the best-fitting nonlinear dose-response curve model. The 'mselect' function was used to determine the fittest nonlinear model for the fungal mycelial growth inhibition dose response curves. The tested nonlinear models include: the three-parameter log-logistic model (LL.3), the four-parameter loglogistic model (LL.4), the four-parameter Weibull models (W2.4), the Cedergreen-Ritz-Streibig model (CRS.4a), and the Brain-Cousens hormesis models (BC.4). The best-fitting model was determined based on the lowest Akaike's Information Criterion (AIC) value.

Determination of baseline $\mathrm{EC}_{50}$ values - conidia germination inhibition assay. Macroconidia were plated on fungicide amended agar medium to determine the inhibitory effect of fluopyram on $F$. virguliforme spore germination. A conidia spore suspension was prepared from a 6-day-old $F$. virguliforme colony grown on PDA by collecting conidia from $F$. virguliforme sporodochia. Sterilized water $(5 \mu \mathrm{l})$ was added to a sporodochium and a pipette was used to homogenize the water and conidia by pipetting up and down, and a 5 - $\mu 1$ condial suspension was collected and diluted into 1-ml sterilized water in a 1.5-ml eppendorf tube. Technical grade fluopyram (Bayer CropScience) was dissolved in dimethyl sulfoxide (DMSO) to make a stock solution for making medium. Based on a preliminary experiment, five fluopyram final concentrations $(0.1,1,5,10$, and $20 \mu \mathrm{g} / \mathrm{ml}$ including a $0-\mu \mathrm{g} / \mathrm{ml}$ control, i.e., DMSO was added to the

Table 2. $\mathrm{EC}_{50}$ of $F$. virguliforme isolates used in the conidia germination inhibition assay testing against SDHI fungicide fluopyram. Isolates were collected from seven states in the United States from 2009 to $2014^{\mathrm{a}}$

\begin{tabular}{lrll}
\hline State & $\mathbf{N}$ & \multicolumn{1}{c}{ Years } & $\mathbf{E C}_{\mathbf{5 0}} \mathbf{b}(\boldsymbol{\mu g} \mathbf{g} \mathbf{m l})$ \\
\hline Arkansas & 3 & $2012-2013$ & $1.24-2.94$ \\
Illinois & 48 & $2010-2014$ & $0.81-5.58^{*}$ \\
Indiana & 3 & 2013 & $1.30-2.18$ \\
Iowa & 5 & 2013 & $2.18-4.07$ \\
Kansas & 1 & 2012 & 3.31 \\
Kentucky & 1 & 2014 & 2.54 \\
Michigan & 10 & $2009-2012$ & $1.34-4.08$ \\
Minnesota & 1 & Unknown & 3.41 \\
Unknown $^{c}$ & 3 & 2013 & $1.57-2.38$ \\
Total & $75^{*}$ & & \\
\hline
\end{tabular}

a An asterisk (*) indicates values that do not include the isolate from Illinois $(\mathrm{n}=1)$ that did not reach $50 \%$ growth inhibition at the highest concentration of fluopyram $(100 \mu \mathrm{g} / \mathrm{ml})$.

${ }^{\mathrm{b}}$ Range of $\mathrm{EC}_{50}$ estimation for the isolates from each state.

${ }^{\mathrm{c}}$ Indicates isolates with unknown source of origin. 
control) were used in the conidia germination experiment. A conidia suspension $(20 \mu \mathrm{l})$ was transferred and evenly spread with a bent glass rod onto PDA amended with different concentrations of fluopyram. Cultures were incubated in ambient light at $20^{\circ} \mathrm{C}$ for $20 \mathrm{~h}$. After incubation, 50 conidia on two replicate plates were evaluated under a microscope to determine the conidia germination rate. A conidium in which the germination tube grew to the length of the conidium was counted as germinated.

Data analyses. The effective fungicide concentration to reduce mycelial growth or conidia germination rate by $50 \%\left(\mathrm{EC}_{50}\right)$ for the isolates were calculated by fitting the mycelial relative growth rate against the log transformed fungicide concentrations using the best fitting model (LL.4) in the R package "drc" v2.5 (Ritz and Streibig 2005). The calculated $\mathrm{EC}_{50}$ values were filtered through two thresholds: (i) relative growth inhibition at the highest fluopyram concentration was more than $50 \%$ of the zero-control; (ii) $P$ value of the $\mathrm{EC}_{50}$ parameter estimation less than 0.05. ANOVA was performed to determine the significance level with the "car" package using type II sum of squares to determine the sources of variances in $\mathrm{EC}_{50}$ values comparisons. Figures were prepared with the "ggplot2" package (Wickham 2009) in $\mathrm{R}$. Estimated $\mathrm{EC}_{50}$ values of all the isolates are listed in Supplementary Tables S1 and S2. The R scripts used for data analysis were made accessible through Zenodo (doi:10.5281/zenodo.53939).

Comparison of the mycelial and spore germination assays. To compare the fungicide sensitivity testing methods, 20 isolates were selected for testing with both methods (Fig. 1). To determine the differences between these two methods, $\mathrm{EC}_{50}$ values calculated for each of these methods were compared using a paired $t$ test, and the $\mathrm{EC}_{50}$ mean contrasts were plotted in a forest plot using $\mathrm{R}$ package "latticeExtra" (Sarkar 2008). The $\mathrm{EC}_{50}$ values calculated using these two methods were regressed over each other in a linear model, and the Spearman correlation was used to determine the level of correlation significance using base R package "stats" (R Core Team 2015).

\section{Results}

Model selection and data validation for the mycelial growth inhibition assay. The four-parameter-log-logistic model (LL.4) was determined to be the best-fitting model based on the lowest AIC for most of the isolates (78\%) tested in the mycelial growth inhibition assay (Fig. 2). The remaining $22 \%$ of the isolates in the mycelial growth assay showed a hormetic effect, where the mycelial growth rate was higher in the low-concentration fungicide treatment $(1 \mu \mathrm{g} / \mathrm{ml})$ than the nonfluopyram control treatment. The models specialized to describe the "u-shape" hormesis were included in the model selection, and the Brain-Cousens modified log-logistic model (BC.4) was determined to be the best-fitting model for the hormetic effect isolates. To keep a parsimonious summary for $\mathrm{EC}_{50}$ calculation, the four-parameter log-logistic model was used consistently for all the mycelial growth inhibition assay data.

To validate the robustness of the mycelial growth inhibition assays, two $F$. virguliforme isolates were randomly selected from each of the 11 batch runs to repeat the mycelial growth inhibition assay. The estimation of $\mathrm{EC}_{50}$ using mycelial growth inhibition was reproducible, as $\mathrm{EC}_{50}$ value replicates calculated for the 21 out of 22 had means confidence intervals that overlapped the repeat means.

Mycelial growth sensitivity against fluopyram. In the mycelial growth inhibition assay, 116 out of $130 \mathrm{~F}$. virguliforme isolates demonstrated mycelial growth inhibition by $50 \%$ on the medium amended with fluopyram. Eight isolates $(6.1 \%)$ were only inhibited by between 0 and $43.8 \%$ at the highest fluopyram concentration of $100 \mu \mathrm{g} / \mathrm{ml}$; five isolates were filtered at the nonlinear regression step; and one isolate failed at the image analysis step possibly due to heavily pigmented agar-medium background. The calculated $\mathrm{EC}_{50}$ values for 116 isolates ranged from 1.53 to $9.28 \mu \mathrm{g} / \mathrm{ml}$, with mean and median $\mathrm{EC}_{50}$ of 3.35 and $3.25 \mu \mathrm{g} / \mathrm{ml}$, respectively (Fig. 3). $\mathrm{EC}_{50}$ values for most isolates $(78.5 \%)$ were within a range between 2 and $4 \mu \mathrm{g} / \mathrm{ml}$. There were no significant differences in $\mathrm{EC}_{50}$ values among the isolates collected from five states $(P=0.41)$ in the U.S. (Table 1). The frequency distribution of $\mathrm{EC}_{50}$ values for the 116 isolates was a unimodal curve, with a right tail distribution indicating the presence of a few $F$. virguliforme isolates less sensitive to fluopyram, not including those 14 isolates for which we could not calculate an $\mathrm{EC}_{50}$ (Fig. 3).

Mycelial growth hormetic effects. A hormetic effect was solely observed in the mycelial growth inhibition assay at the concentrations of $1 \mu \mathrm{g} / \mathrm{ml}$ and $0.5 \mu \mathrm{g} / \mathrm{ml}$ (data not shown) in the fluopyram amended medium. Twenty-two percent $(n=29)$ of the isolates tested in the mycelial growth inhibition assay demonstrated a hormetic effect. The best-fitting model for these isolates was determined to be the Brain-Cousens hormesis model (BC.4) based on the lowest AIC values (Fig. 4, Table 3). The calculated $\mathrm{EC}_{50}$ values with the BC. 4 model were significantly higher than the $\mathrm{EC}_{50}$ values calculated using the LL.4 model ( $P=0.006$, Table 3$)$. On average, the BC. 4 model estimated $\mathrm{EC}_{50}$ values were higher than the LL. 4 model estimated $\mathrm{EC}_{50}$ values by $10 \mu \mathrm{g} / \mathrm{ml}$. Between these two models, 22 out of $29 \mathrm{EC}_{50}$ values estimated using the BC.4 model fell within the concentration range where the $50 \%$ growth inhibition was reached, while three of the $29 \mathrm{EC}_{50}$ values estimated using the LL. 4 model fell
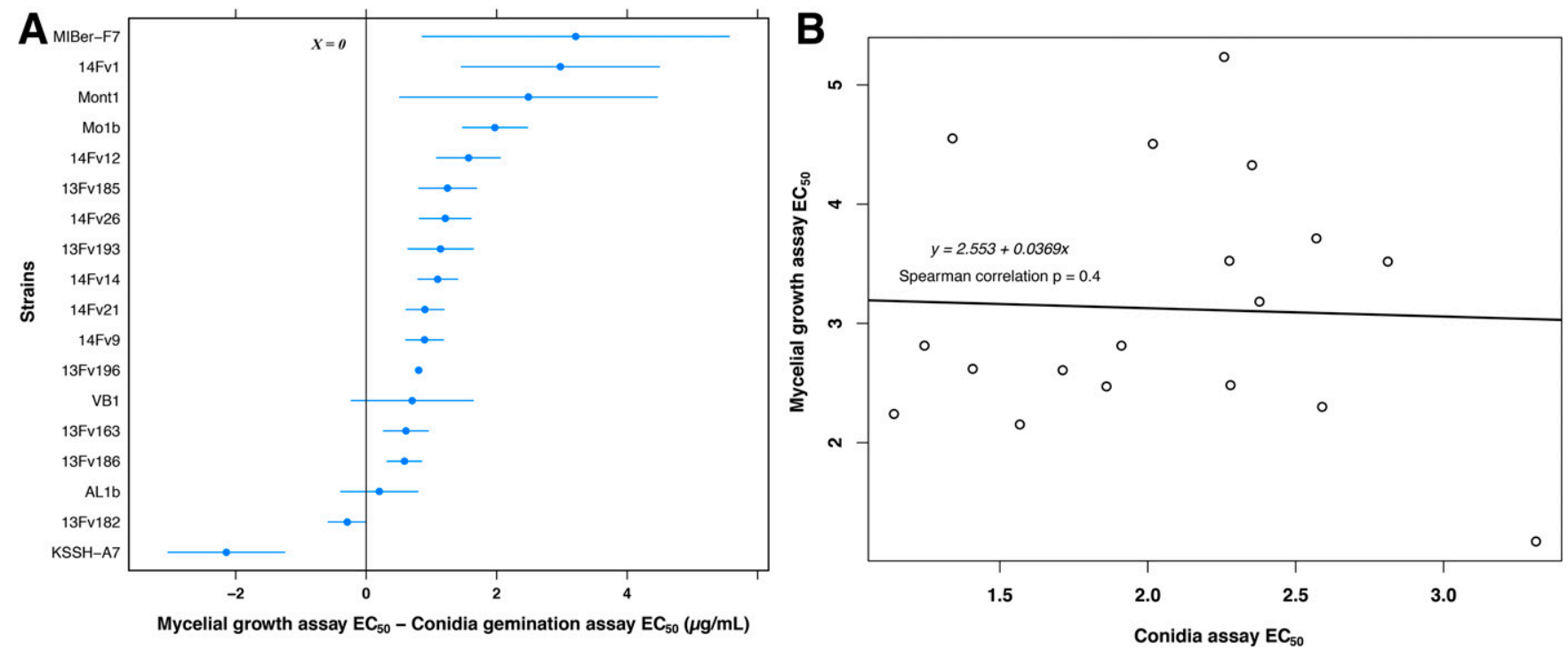

Fig. 1. Comparison of the difference in $\mathrm{EC}_{50}$ values estimated using mycelial growth inhibition assay $(\mathbf{A})$ and conidia germination inhibition assay (B). The contrast differences between the mycelial growth inhibition and conidia germination assay was plotted for $20 \mathrm{~F}$. virguliforme isolates $(\mathrm{A})$. The $\mathrm{EC}_{50}$ values calculated using both methods were correlated using the general linear model, but no significant correlation was observed between these two methods (Spearman correlation $P=0.40$ ). 
within the concentration range where the $50 \%$ growth inhibition was reached. Neither of these two models fell within the concentration range where $50 \%$ growth inhibition was reached for the remaining four isolates (Table 3). Overall, the LL.4 model underestimated the $\mathrm{EC}_{50}$ for the isolates showing hormetic effects, and BC.4 had a more accurate estimation of $\mathrm{EC}_{50}$ for the isolates showing a hormetic effect.

Model selection and data validation for the conidia germination inhibition assay. For the conidia germination assay, conidia germination rate decreased as fluopyram concentrations increased. The best-fitting model selected for the conidia germination assay dose response data were the four-parameter-log-logistic (LL.4) model and less than $10 \%$ of the isolates showed a better fit with other nonlinear models (e.g., Weibull, Cedergreen-Ritz-Streibig model, or
Brain-Cousens hormesis models). To keep a parsimonious summary for $\mathrm{EC}_{50}$ and to be able to compare among isolates, the LL.4 model was used for all isolates (Fig. 2).

To validate the reproducibility of the conidia germination inhibition assay, 51 isolates were tested in two separate runs to compare their $\mathrm{EC}_{50}$ estimations. The confidence intervals of the $\mathrm{EC}_{50}$ estimation were calculated for the replicated isolates, and 40 out of 51 replicated isolates were determined to be not significantly different between two replicates, as indicated by the overlapped $\mathrm{EC}_{50}$ mean confidence interval (Supplementary Figure S2).

Conidia germination sensitivity against fluopyram. Most of the $F$. virguliforme isolates were sensitive to fluopyram in the conidia germination inhibition assay. In total, 74 out of $75 \mathrm{~F}$. virguliforme isolates showed a conidia germination rate reduction by $50 \%$ on

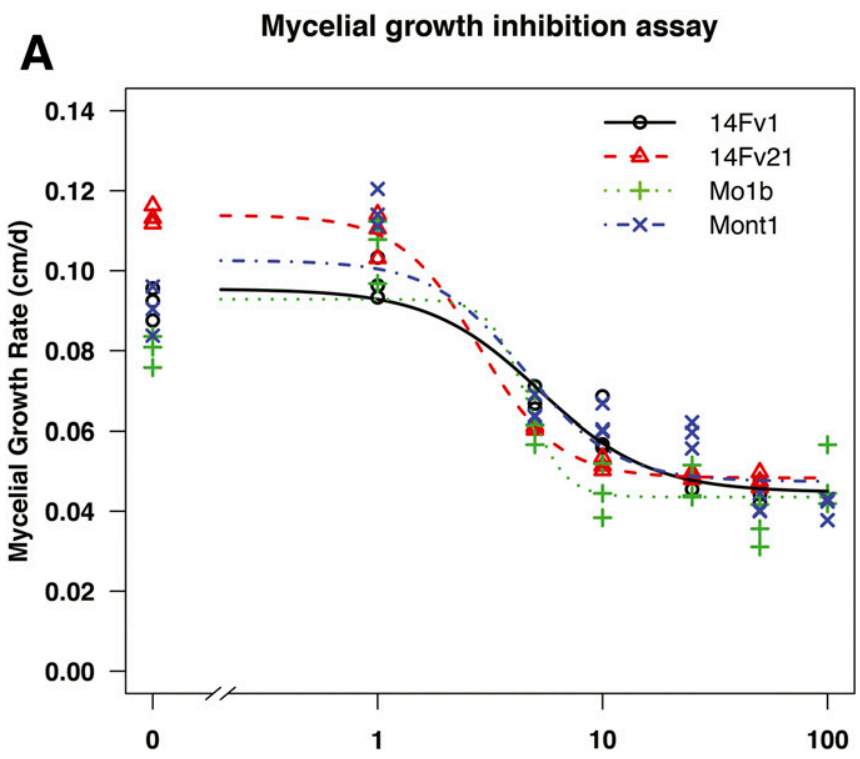

B

\section{Conidial germination assay}

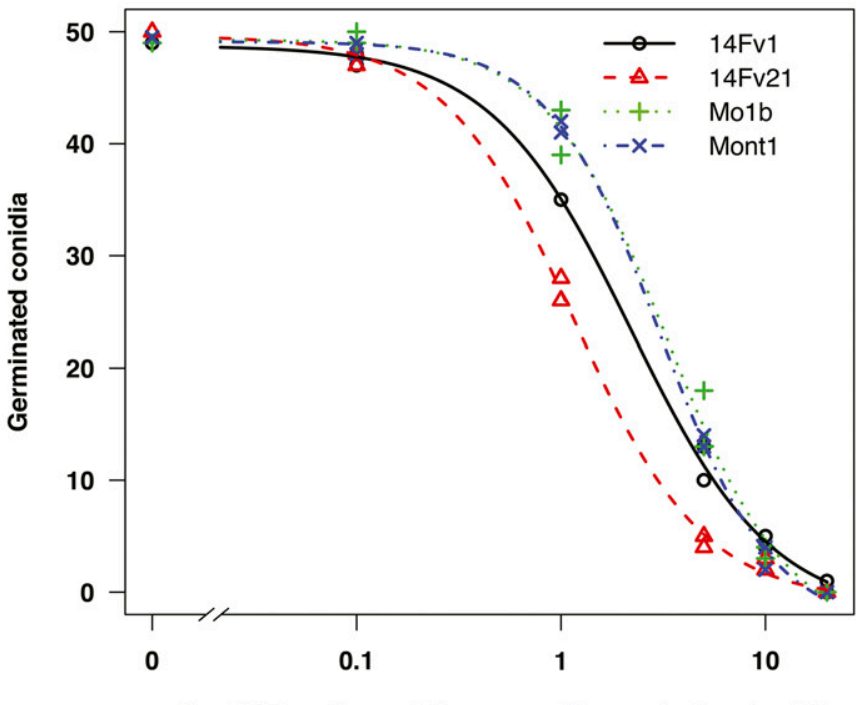

Fig. 2. Dose response curve fitting with the four-parameter log-logistic model for mycelial growth (A) and conidia germination (B) at different concentrations of fluopyram amended in the agar medium for the $F$. virguliforme isolates: 14Fv1, 14Fv21, Mo1b, and Mont1, as represented by "o", " $\Delta$ ", "+", and " $x$ " symbols, respectively.
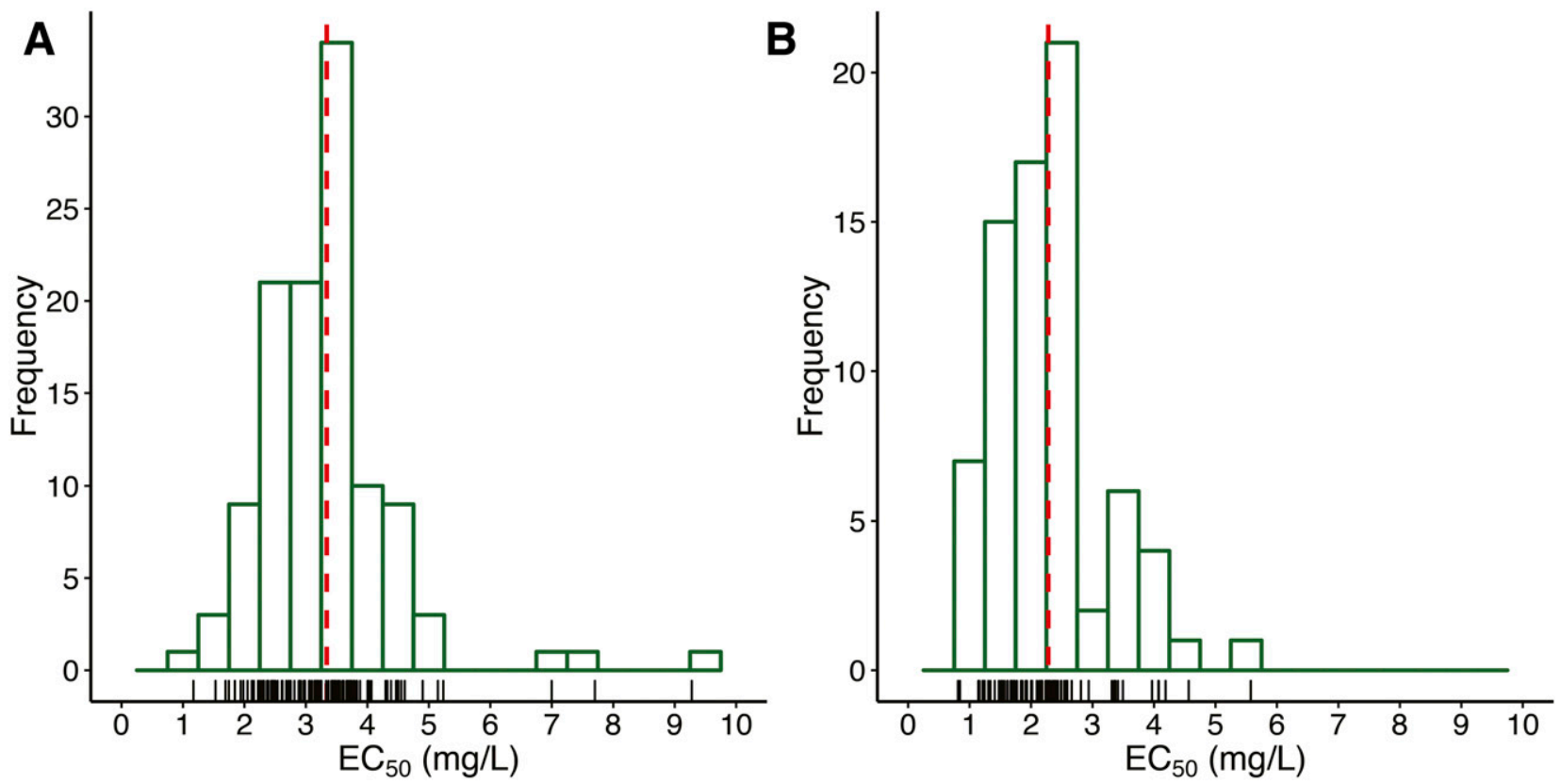

Fig. 3. Frequency distribution of effective fungicide concentration that inhibit growth by $50 \%$ for both mycelial growth inhibition assay (A) and conidia germination inhibition assay (B). The mean $\mathrm{EC}_{50}$ value was indicated as the dotted vertical lines with the mean $\mathrm{EC}_{50}$ values: 3.35 and $2.28 \mu \mathrm{g} / \mathrm{ml}$ for the mycelial growth inhibition assay and conidia germination assay, respectively. 


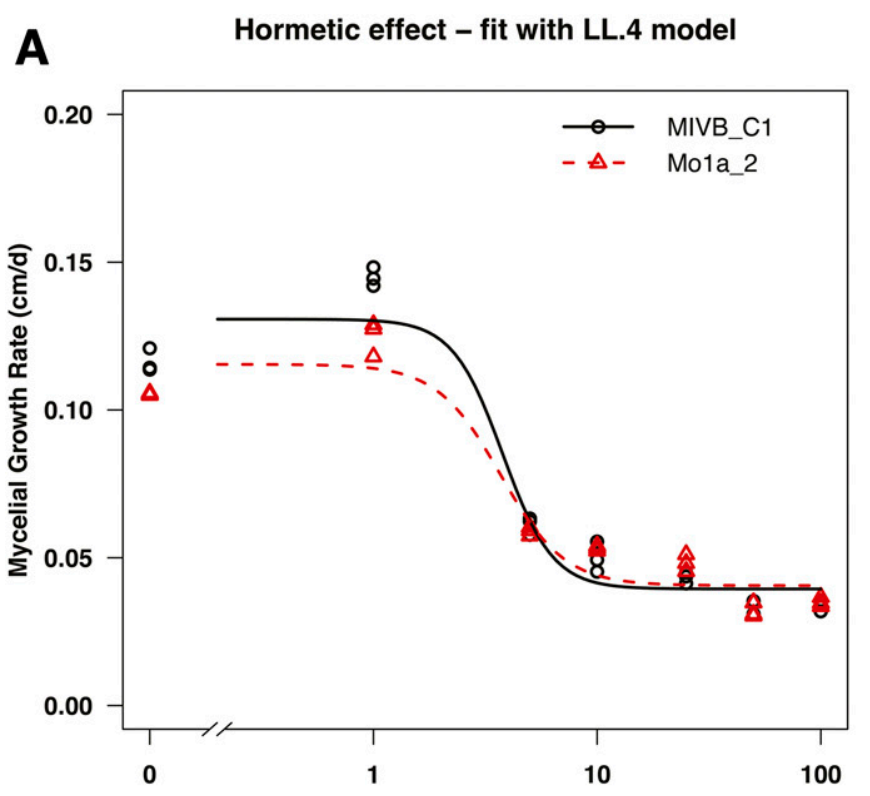

Log10 Transformed fluopyram concentrations $(\mathrm{mg} / \mathrm{L})$
B Hormetic effect - fit with BC.4 model

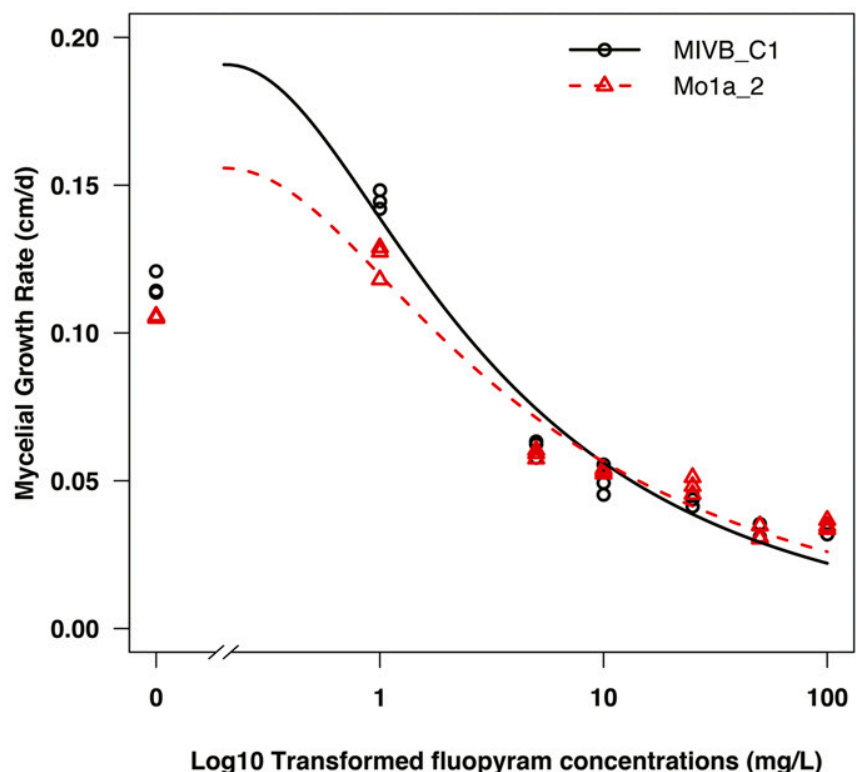

Fig. 4. Dose response curve fitting the isolates showed hormetic effect using the four-parameter logistic model (LL.4) (A) and Brain-Cousens model (BC.4) (B). The hormetic effect isolates showed faster growth rate at $1 \mu \mathrm{g} / \mathrm{ml}$ concentration than the zero-control. The nonlinear regression BC. 4 model $(\mathrm{AIC}=-271)$ fits better than the LL.4 (AIC $=-259)$ model for the isolates that showed hormesis.

Table 3. Comparison of $\mathrm{EC}_{50}$ values calculated using the four-parameter log-logistic model (LL.4) and the Brain-Cousens model (BC.4) for the $F$. virguliforme isolated that showed hormetic effect in the mycelial growth inhibition assay. At model selection, the AIC values calculated for LL.4 and BC. 4 models were -4148 and -3926 (lower is better), respectively

\begin{tabular}{|c|c|c|c|c|c|c|c|c|c|c|c|}
\hline \multirow[b]{2}{*}{ Strain } & \multirow[b]{2}{*}{ Set $^{\mathbf{a}}$} & \multicolumn{2}{|c|}{ LL.4 } & \multicolumn{2}{|c|}{ BC.4 } & \multirow[b]{2}{*}{ Difference in $\mathbf{E C}_{\mathbf{5 0}}$} & \multicolumn{4}{|c|}{ Relative growth ratio ${ }^{\mathrm{e}}$} & \multirow[b]{2}{*}{ Better Model $^{\mathrm{d}}$} \\
\hline & & $\overline{\mathbf{E C}_{50}}$ & $\overline{\text { StdErrb }^{b}}$ & $\mathbf{E C}_{50}$ & $\overline{\text { StdErr }}$ & & $5 \mu \mathrm{g} / \mathrm{ml}$ & $10 \mu \mathrm{g} / \mathrm{ml}$ & $50 \mu \mathrm{g} / \mathrm{ml}$ & $100 \mu \mathrm{g} / \mathrm{ml}$ & \\
\hline AL1a & 2 & 3.10 & 0.49 & 5.14 & 0.60 & 2.04 & 0.45 & 0.38 & 0.22 & $-f$ & LL.4 \\
\hline ARLE-A1 & 1 & 3.73 & 0.98 & 5.77 & 1.13 & 2.04 & 0.47 & 0.44 & 0.18 & - & LL.4 \\
\hline Mo4a & 1 & 2.91 & 0.74 & 5.17 & 0.97 & 2.26 & 0.45 & 0.38 & 0.22 & - & LL.4 \\
\hline MIVB-A5 & 2 & 1.21 & 0.11 & 3.67 & 0.41 & 2.47 & 0.51 & 0.35 & 0.25 & 0.30 & None \\
\hline MIVB-C1 & 10 & 3.79 & 0.50 & 9.14 & 1.47 & 5.35 & 0.53 & 0.43 & 0.29 & 0.29 & BC. 4 \\
\hline MITU-B1 & 12 & 4.01 & 1.46 & 9.98 & 2.46 & 5.97 & 0.54 & 0.49 & 0.32 & 0.33 & BC. 4 \\
\hline MISTJ-A1 & 3 & 4.12 & 1.03 & 10.44 & 4.13 & 6.32 & 0.55 & 0.43 & 0.26 & 0.38 & None \\
\hline STJ-7P2ss & 1 & 3.59 & 1.18 & 9.93 & 3.66 & 6.33 & 0.54 & 0.47 & 0.33 & - & BC. 4 \\
\hline MIBer-E6 & 3 & 3.74 & 0.79 & 11.13 & 3.78 & 7.39 & 0.49 & 0.43 & 0.23 & 0.34 & None \\
\hline Mo1a-2 & 10 & 3.71 & 0.50 & 12.21 & 2.18 & 8.50 & 0.56 & 0.51 & 0.30 & 0.33 & BC. 4 \\
\hline MIBer-F3 & 10 & 3.75 & 0.55 & 13.38 & 2.78 & 9.63 & 0.57 & 0.52 & 0.30 & 0.38 & BC. 4 \\
\hline KSSH-G2 & 10 & 3.73 & 0.61 & 14.66 & 3.03 & 10.93 & 0.58 & 0.52 & 0.36 & 0.36 & BC. 4 \\
\hline MISA-A1 & 7 & 4.46 & 1.12 & 20.16 & 5.09 & 15.69 & 0.64 & 0.58 & 0.36 & 0.38 & BC. 4 \\
\hline Mo3a & 9 & 4.38 & 0.92 & 23.83 & 6.94 & 19.45 & 0.69 & 0.59 & 0.38 & 0.42 & BC. 4 \\
\hline INMO-A6 & 10 & 4.47 & 0.82 & 26.09 & 5.92 & 21.62 & 0.70 & 0.60 & 0.39 & 0.43 & BC. 4 \\
\hline MIVB-A6 & 1 & 4.61 & 1.58 & 27.38 & 11.10 & 22.77 & 0.79 & 0.68 & 0.47 & - & BC. 4 \\
\hline Mont1 & 14 & 9.34 & 2.00 & 34.10 & 7.64 & 24.76 & 0.77 & 0.75 & 0.43 & 0.45 & BC. 4 \\
\hline MISTJ-F6 & 11 & 4.50 & 1.06 & 30.45 & 8.89 & 25.96 & 0.75 & 0.67 & 0.43 & 0.49 & BC. 4 \\
\hline 14Fv1 & 13 & 5.24 & 0.87 & 33.39 & 6.49 & 28.16 & 0.74 & 0.66 & 0.49 & - & BC. 4 \\
\hline MISTJ-E4 & 11 & 4.00 & 1.48 & 36.19 & 13.25 & 32.19 & 0.59 & 0.54 & 0.42 & 0.42 & BC. 4 \\
\hline Mont1 & 7 & 4.51 & 1.39 & 42.01 & 11.40 & 37.51 & 0.77 & 0.75 & 0.43 & 0.45 & BC. 4 \\
\hline $\mathrm{Ca} 2 \mathrm{a}$ & 4 & 4.30 & 1.66 & 117.88 & 96.28 & 113.58 & 0.87 & 0.70 & 0.64 & 0.72 & BC. $4^{\mathrm{g}}$ \\
\hline MIBer-E4 & 8 & 7.70 & 3.00 & 231.42 & 170.19 & 223.72 & 1.11 & 1.00 & 0.69 & 0.81 & BC. $4^{\mathrm{g}}$ \\
\hline ARLE-C3a & 11 & $\mathrm{NA}^{\mathrm{c}}$ & NA & 270.18 & 187.66 & NA & 1.09 & 1.00 & 0.70 & 0.62 & BC. $4 \mathrm{~g}$ \\
\hline KSSH-A4 & 1 & $\mathrm{NA}^{\mathrm{c}}$ & NA & 11.46 & 4.18 & NA & 0.53 & 0.51 & 0.38 & - & BC. 4 \\
\hline LE-11-1 & 3 & $\mathrm{NA}^{\mathrm{c}}$ & NA & 154.55 & 130.28 & NA & 1.01 & 0.92 & 0.59 & 0.76 & BC. $4^{\mathrm{g}}$ \\
\hline MIBer-A5 & 11 & $\mathrm{NA}^{\mathrm{c}}$ & NA & 22.29 & 9.16 & NA & 0.61 & 0.51 & 0.43 & 0.39 & BC. 4 \\
\hline MIBer-D1 & 2 & $\mathrm{NA}^{\mathrm{c}}$ & NA & 7.78 & 1.37 & NA & NA & 0.43 & NA & - & BC. 4 \\
\hline MITU-B1 & 2 & $\mathrm{NA}^{\mathrm{c}}$ & NA & 11.85 & 3.76 & NA & 0.54 & 0.49 & 0.32 & 0.33 & None \\
\hline
\end{tabular}

${ }^{\mathrm{a}}$ Indicates batch number for different sets of testing isolates.

b Indicates standard error.

c Indicates that the $\mathrm{EC}_{50}$ parameter estimation in the LL.4 model does not have a significant $P$ value.

${ }^{\mathrm{d}}$ Indicates $\mathrm{EC}_{50}$ estimated using either LL.4 or BC.4 falls within the range of relative growth inhibition by $50 \%$.

e Relative germination ratio at these concentrations to the $0 \mu \mathrm{g} / \mathrm{ml}$ control, the percentage values lower than $50 \%$ are in bold font.

${ }^{\mathrm{f}}$ Indicates missing data.

${ }^{\mathrm{g}} \mathrm{EC}_{50}$ estimation out of tested concentration range. 
medium amended with fluopyram, with one isolate not reaching a 50\% conidia germination rate reduction even at the highest fluopyram concentration $(20 \mu \mathrm{g} / \mathrm{ml})$. The calculated $\mathrm{EC}_{50}$ values for the 74 isolates ranged between 0.81 and $5.57 \mu \mathrm{g} / \mathrm{ml}$, with mean and median $\mathrm{EC}_{50}$ of 2.28 and $2.24 \mu \mathrm{g} / \mathrm{ml}$, respectively (Fig. 3). $\mathrm{EC}_{50}$ values for most of the isolates (89\%) fell within a range of 1 and $3.5 \mu \mathrm{g} / \mathrm{ml}$ with a right-tailed unimodal distribution indicating that most isolates were sensitive to fluopyram, not including one isolate for which we could not calculate $\mathrm{EC}_{50}$. There appeared to be no difference in sensitivity of $F$. virguliforme isolates between states (Table 2).

Differences between two fungicide sensitivity testing methods. The $\mathrm{EC}_{50}$ values estimated using the mycelial growth inhibition assay were significantly higher than the $\mathrm{EC}_{50}$ values calculated with the conidia germination assay $(P<0.01)$. Of the 20 cross-validated $F$. virguliforme isolates, 15 isolates showed higher $\mathrm{EC}_{50}$ estimates for the mycelial growth inhibition assay than the conidia germination inhibition assay (Fig. $1 ; P<0.01$ ). Between the $\mathrm{EC}_{50}$ values calculated from both of the fungicide sensitivity testing methods, there was no statistically significant correlation between those two sets of $\mathrm{EC}_{50}$ estimation data (Spearman correlation was nonsignificant at $P=0.4$, Fig. 1).

\section{Discussion}

Most of the isolates tested in this study were sensitive to fluopyram, but a small fraction of isolates $(\sim 5 \%)$ were insensitive. The presence of fluopyram-insensitive $F$. virguliforme isolates may imply the risk of accumulating less-sensitive isolates in field populations, and result in possible disease management failure. Based on this survey, there is no significant difference in $\mathrm{EC}_{50}$ values among sampling locations or years of collections, which is perhaps expected since this pathogen was most likely not previously exposed to fluopyram. Although one of the major fluopyram products, Luna Privilege, is registered on horticultural crops that could have been in rotation with soybean, most of the $F$. virguliforme isolates in this study were collected from fields predominantly in corn-soybean rotations. Additionally, other SDHI fungicides may have been applied in soybean seed treatments, but fluopyram binds to a different cavity than the other SDHIs, possibly reducing cross-resistance (Fraaije et al. 2012).

There is a fundamental difference in fungicide sensitivity tests between the mycelial growth inhibition assay and the conidia germination inhibition assay, including starting fungal materials and experiment durations. Fungicide sensitivity $\mathrm{EC}_{50}$ values evaluated using the mycelial growth inhibition assay were significantly higher than the fungicide sensitivity $\mathrm{EC}_{50}$ values calculated using the conidia germinationinhibition assay by $1 \mu \mathrm{g} / \mathrm{ml}$. The difference in $\mathrm{EC}_{50}$ estimation using different methods was reported in a study for which Alternaria alternata isolates were tested against the SDHI fungicide boscalid (Vega and Dewdney 2015). Contrary to the Vega and Dewdney (2015) findings that mycelial growth of A. alternata was more sensitive to boscalid than conidia germination, in this study fluopyram was more effective in inhibiting $F$. virguliforme conidia germination than mycelia growth. The difference in $\mathrm{EC}_{50}$ estimations from these two methods could be explained by a fundamental difference in transferring of starting materials: conidia and mycelia. Before transferring to the fungicide amended agar medium, the conidia were in a "dormant" condition, while the mycelia were taken from the actively growing part of a colony (Gougouli and Koutsoumanis 2013). The different onset of metabolic levels (Cochrane and Cochrane 1966; Liu et al. 2015) may affect the dose response to fungicide treatment. Thus, it may not be surprising to observe differences in $\mathrm{EC}_{50}$ estimations using these two methods. A combination of these two testing methods provided a relatively complete estimation of $F$. virguliforme sensitivity against the fluopyram fungicide.

At the lower fungicide-concentration treatment, a hormetic effect was observed for $22 \%$ of the isolates solely in the mycelial growth inhibition assay. The presence of a hormetic effect on isolates in the field indicate that exposure to sublethal doses of fungicide can possibly result in more severe disease symptoms (Garzon et al. 2011). In this study, the hormetic effect was observed at concentrations of 0.5 and $1 \mu \mathrm{g} / \mathrm{ml}$ (concentration $0.5 \mu \mathrm{g} / \mathrm{ml}$ was tested in the preliminary study; data not shown), indicating that the application of fluopyram to seed should ensure an effective concentration above $1 \mu \mathrm{g} / \mathrm{ml}$. Additional studies including inoculated field or greenhouse trials could be conducted to compare the effect of fluopyram and rate of product on soybean infection and disease management against a subset of $F$. virguliforme isolates that demonstrate hormesis and those that do not demonstrate the hormesis effect. Dilution of fluopyram in soil or plant tissue may have a significant role in the behavior of hormetic isolates.

Multiple nonlinear models were selected to fit the fungi growth dose-response curve. The four-parameter log-logistic model (LL.4) was determined to be the best-fitting model based on the Akaike's information criteria for most of the isolates $(78 \%)$. However, the best fitting model for isolates demonstrating a hormetic effect was the Brain-Cousens hormesis (BC.4) model, which was specifically designed for the hormetic effect dose-response curve (Cedergreen et al. 2005). The BC. 4 model showed a higher estimation of $\mathrm{EC}_{50}$ than the LL. 4 model because the hormesis model will lead to higher $\mathrm{EC}_{50}$ levels, as hormetic effect could possibly delay the onset of toxicity. In reality, most $\mathrm{EC}_{50}$ values (22 out of 29) estimated using the BC. 4 fell within the concentration range where $50 \%$ growth inhibition was reached (Table 3). The LL.4 model underestimated the $\mathrm{EC}_{50}$ for the isolates demonstrating hormesis. Although the BC.4 performs better in predicting $\mathrm{EC}_{50}$ for the isolates with a hormetic effect, to achieve the most parsimonious interpretation of the $\mathrm{EC}_{50}$ estimations (C. Ritz, personal communication), the LL.4 model was used consistently for all the isolates.

A small fraction of $F$. virguliforme isolates were determined to be less sensitive to fluopyram treatment in the in vitro assay. Based on the FRAC definition, $\mathrm{EC}_{50}$ is the dose that provides $50 \%$ inhibition of the isolates as compared with a non-fungicide-amended control, which is also known as absolute $\mathrm{EC}_{50}$. To calculate the $\mathrm{EC}_{50}$, isolates that showed less than $50 \%$ growth inhibition at the highest fungicide concentration treatment were filtered out from the data set before using the data for subsequent analysis. In this study, the less sensitive isolates tend to be the slow-growing isolates which secreted melaninlike pigments in the medium, even in the absence of fluopyram. Similar results of reduced fungicide sensitivity coupled with slow growth rate and secretion of melanin, were also reported for other plant or human pathogens, such as Zymoseptoria tritici (Lendenmann et al. 2015) and Paracoccidioides brasiliensis (Taborda et al. 2008). The presence of less sensitive isolates does not always indicate a rapid accumulation of less sensitive isolates in the field. Currently, fluopyram for use in soybeans has been applied as a seed treatment, which is only applied once per season and results in very localized exposure of the fungicide. Therefore, the selection pressure to accumulate resistant isolates may not be as strong as it is for the management of foliar diseases, where chemistry is broadly applied to a field and application may occur multiple times per season.

Besides $F$. virguliforme, SDS can be caused by three additional Fusarium species (F. tucumaniae, F. brasiliense, and F. crassistipitatum) in South America, which are phylogenetically clustered within the clade-2 Fusarium solani species complex (FSSC) with $F$. virguliforme (Aoki et al. 2005; Aoki et al. 2012a). Also, there are additional Fusarium species within the clade- 2 FSSC causing root rots on dry bean, and these Fusarium species are also phylogenetically close to $F$. virguliforme (Aoki et al. 2012b). Therefore, it is possible that fluopyram will also be effective in inhibiting mycelial growth or conidia germination for those Fusarium species. The data generated from the in vitro assays conducted in this study enable future monitoring efforts for changes in $F$. virguliforme sensitivity to fluopyram.

\section{Acknowledgments}

We thank Dr. Tyre Proffer, Dr. Alejandro Rojas, and Janette Jacobs for their suggestions on the design of this experiment. We also thank Zachary Noel for his technical suggestions for data analysis. The set of NRRL $F$. virguliforme isolates was kindly provided by ARS culture collection, USDA, Peoria IL. We also thank Dr. Leonor Leandro for sharing some of the $F$. virguliforme isolates. This work was supported by Bayer CropSciences, the North Central Soybean Research Program (NCSRP), and the Michigan Soybean Promotion Committee. Collection of some of the $F$. virguliforme isolates was supported by Agriculture and Food Research Initiative Competitive Grant no. 2013-68004-20374 from the USDA National Institute of Food and Agriculture. 


\section{Literature Cited}

Amiri, A., Heath, S. M., and Peres, N. A. 2014. Resistance to fluopyram, fluxapyroxad, and penthiopyrad in Botrytis cinerea from strawberry. Plant Dis. 98:532-539.

Aoki, T., O’Donnell, K., and Scandiani, M. M. 2005. Sudden death syndrome of soybean in South America is caused by four species of Fusarium: Fusarium brasiliense sp. nov., F. cuneirostrum sp. nov., $F$. tucumaniae, and $F$. virguliforme. Mycoscience 46:162-183.

Aoki, T., Scandiani, M. M., and O’Donnell, K. 2012a. Phenotypic, molecular phylogenetic, and pathogenetic characterization of Fusarium crassistipitatum sp. nov., a novel soybean sudden death syndrome pathogen from Argentina and Brazil. Mycoscience 53:167-186.

Aoki, T., Tanaka, F., Suga, H., Hyakumachi, M., Scandiani, M. M., and O'Donnell, K. 2012b. Fusarium azukicola sp nov., an exotic azuki bean rootrot pathogen in Hokkaido, Japan. Mycologia 104:1068-1084.

Avenot, H., Morgan, D. P., and Michailides, T. J. 2008. Resistance to pyraclostrobin, boscalid and multiple resistance to Pristine (R) (pyraclostrobin plus boscalid) fungicide in Alternaria alternata causing alternaria late blight of pistachios in California. Plant Pathol. 57:135-140.

Avenot, H. F., and Michailides, T. J. 2010. Progress in understanding molecular mechanisms and evolution of resistance to succinate dehydrogenase inhibiting (SDHI) fungicides in phytopathogenic fungi. Crop Prot. 29:643-651.

Bradley, C. A., and Pedersen, D. K. 2011. Baseline sensitivity of Cercospora zeae-maydis to quinone outside inhibitor fungicides. Plant Dis. 95:189-194.

Broomfield, P. L. E., and Hargreaves, J. 1992. A single amino-acid change in the iron-sulphur protein subunit of succinate dehydrogenase confers resistance to carboxin in Ustilago maydis. Curr. Genet. 22:117-121.

Cedergreen, N., Ritz, C., and Streibig, J. C. 2005. Improved empirical models describing hormesis. Environ. Toxicol. Chem. 24:3166-3172.

Chiocchio, V., Venedikian, N., Martinez, E. A., Menendez, A., Ocampo, A. J., and Godeas, A. 2000. Effect of the fungicide benomyl on spore germination and hyphal length of the arbuscular mycorrhizal fungus Glomus mosseae. Int. Microbiol. 3:173-175.

Cochrane, V. W., and Cochrane, J. C. 1966. Spore germination and carbon metabolism in Fusarium solani V. changes in anaerobic metabolism and related enzyme activities during development. Plant Physiol. 41:810-814.

Fraaije, B. A., Bayon, C., Atkins, S., Cools, H. J., Lucas, J. A., and Fraaije, M. W. 2012. Risk assessment studies on succinate dehydrogenase inhibitors, the new weapons in the battle to control Septoria leaf blotch in wheat. Mol. Plant Pathol. 13:263-275.

FRAC. 2015. Fungicide Resistance Action Committee (FRAC) - Fungicides sorted by mode of action. Online publication. http://www.frac.info/publications/downloads

Gao, X., Hartman, G., and Niblack, T. 2006. Early infection of soybean roots by Fusarium solani f. sp glycines. Phytopathology 96:S38.

Garzón, C. D., Molineros, J. E., Yanez, J. M., Flores, F. J., Jimenez-Gasco, M. D., and Moorman, G. W. 2011. Sublethal doses of mefenoxam enhance Pythium damping-off of Geranium. Plant Dis. 95:1233-1238.

Gougouli, M., and Koutsoumanis, K. P. 2013. Relation between germination and mycelium growth of individual fungal spores. Int. J. Food Microbiol. 161: 231-239.

Gunatilleke, I. A. U. N., Arst, H. N., and Scazzocchio, C. 1975. Three genes determine the carboxin sensitivity of mitochondrial succinate oxidation in Aspergillus nidulans. Genet. Res. 26:297-305.

Hartman, G. L., Chang, H. X., and Leandro, L. F. 2015. Research advances and management of soybean sudden death syndrome. Crop Prot. 73:60-66.

Ishii, H., Miyamoto, T., Ushio, S., and Kakishima, M. 2011. Lack of crossresistance to a novel succinate dehydrogenase inhibitor, fluopyram, in highly boscalid-resistant isolates of Corynespora cassiicola and Podosphaera xanthii. Pest Manag. Sci. 67:474-482.

Kandel, Y. R., Wise, K. A., Bradley, C., Chilvers, M. I., Tenuta, A., and Mueller, D. S. 2016. Fungicide and cultivar effects on sudden death syndrome and yield of soybean. Plant Dis. 100:1339-1350.

Kolander, T. M., Bienapfl, J. C., Kurle, J. E., and Malvick, D. K. 2012. Symptomatic and asymptomatic host range of Fusarium virguliforme, the causal agent of soybean sudden death syndrome. Plant Dis. 96:1148-1153.
Kuhn, P. J. 1984. Mode of action of carboxamides. Br. Mycol. Soc. Symp. Ser. 9: 155-183.

Lendenmann, M. H., Croll, D., and McDonald, B. A. 2015. QTL mapping of fungicide sensitivity reveals novel genes and pleiotropy with melanization in the pathogen Zymoseptoria tritici. Fungal Genet. Biol. 80:53-67.

Liang, H. J., Di, Y. L., Li, J. L., You, H., and Zhu, F. X. 2015. Baseline sensitivity of pyraclostrobin and toxicity of SHAM to Sclerotinia sclerotiorum. Plant Dis. 99:267-273.

Liu, H. X., Zhao, X. S., Guo, M. X., Liu, H., and Zheng, Z. M. 2015. Growth and metabolism of Beauveria bassiana spores and mycelia. BMC Microbiol. 15:267.

Mueller, D., Wise, K. A., Dufault, N. S., Bradley, C. A., and Chilvers, M. I. 2013 Fungicides for Field Crops. American Phytopathological Society, St. Paul, MN.

Mueller, T. A., Knake, R. P., and Riggs, J. L. 2011. Control of Fusarium virguliforme (sudden death syndrome) with a seed treatment. Phytopathology 101:S124.

Munkvold, G. P. 2009. Seed pathology progress in academia and industry. Annu. Rev. Phytopathol. 47:285-311.

Navi, S. S., and Yang, X. 2008. Foliar symptom expression in association with early infection and xylem colonization by Fusarium virguliforme (formerly F. solani f. sp. glycines), the causal agent of soybean sudden death syndrome. Plant Health Prog. doi:10.1094/PHP-2008-0222-01-RS.

Navi, S. S., and Yang, X. 2016. Impact of crop residue and corn-soybean rotation on the survival of Fusarium virguliforme a causal agent of Sudden Death Syndrome of soybean. J. Plant Pathol. Microbiol.: 7:330-336.

Njiti, V. N., Suttner, R. J., Gray, L. E., Gibson, P. T., and Lightfoot, D. A. 1997. Rate-reducing resistance to Fusarium solani f. sp. phaseoli underlies field resistance to soybean sudden death syndrome. Crop Sci. 37:132-138.

O’Donnell, K., Sink, S., Scandiani, M. M., Luque, A., Colletto, A., Biasoli, M., Lenzi, L., Salas, G., Gonzalez, V., Ploper, L. D., Formento, N., Pioli, R. N., Aoki, T., Yang, X. B., and Sarver, B. A. 2010. Soybean sudden death syndrome species diversity within North and South america revealed by multilocus genotyping. Phytopathology 100:58-71.

R Core Team. 2015. R: A language and environment for statistical computing. R Foundation for Statistical Computing, Vienna, Austria.

Ritz, C., and Streibig, J. C. 2005. Bioassay analysis using R. J. Stat. Softw. 12: $1-22$.

Sarkar, D. 2008. Lattice: Multivariate Data Visualization with R. Springer Science \& Business Media, Berlin/Heidelberg, Germany.

Saville, A., Graham, K., Grunwald, N. J., Myers, K., Fry, W. E., and Ristaino, J. B. 2015. Fungicide sensitivity of us genotypes of Phytophthora infestans to six oomycete-targeted compounds. Plant Dis. 99:659-666.

Taborda, C. P., da Silva, M. B., Nosanchuk, J. D., and Travassos, L. R. 2008. Melanin as a virulence factor of Paracoccidioides brasiliensis and other dimorphic pathogenic fungi: a minireview. Mycopathologia 165:331-339.

Vega, B., and Dewdney, M. M. 2015. Sensitivity of Alternaria alternata from citrus to boscalid and polymorphism in iron-sulfur and in anchored membrane subunits of succinate dehydrogenase. Plant Dis. 99:231-239.

Vick, C. M., Chong, S. K., Bond, J. P., and Russin, J. S. 2003. Response of soybean sudden death syndrome to subsoil tillage. Plant Dis. 87:629-632.

Wang, J., and Chilvers, M. I. 2016. Development and characterization of microsatellite markers for Fusarium virguliforme and their utility within clade 2 of the Fusarium solani species complex. Fungal Ecol. 20:7-14.

Wang, J., Jacobs, J., and Chilvers, M. 2014. Management of soybean sudden death syndrome by seed treatment with fluopyram. Phytopathology 104 (Suppl.):127.

Wang, J., Jacobs, J. L., Byrne, J. M., and Chilvers, M. I. 2015. Improved diagnoses and quantification of Fusarium virguliforme, causal agent of soybean sudden death syndrome. Phytopathology 105:378-387.

Wang, J., Jacobs, J. L., and Chilvers, M. I. 2013. Temporal dynamics of soybean root colonization by Fusarium virguliforme. Phytopathology 103:S2.157.

Weems, J. D., Haudenshield, J. S., Bond, J. P., Hartman, G. L., Ames, K. A., and Bradley, C. A. 2015. Effect of fungicide seed treatments on Fusarium virguliforme infection of soybean and development of sudden death syndrome. Can. J. Plant Pathol. 37:435-447.

Wickham, H. 2009. ggplot2: Elegant graphics for data analysis. Springer Science $\&$ Business Media, Berlin/Heidelberg, Germany. 